University of Nebraska - Lincoln

DigitalCommons@University of Nebraska - Lincoln

Faculty Publications, Department of Physics and Astronomy

Research Papers in Physics and Astronomy

9-16-2005

\title{
Circular dichroism in classical Coulomb scattering involving bremsstrahlung
}

A. A. Krylovetsky

Voronezh State University

N. L. Manakov

Voronezh State University, manakov@phys.vsu.ru

S. I. Marmo

Voronezh State University

Anthony F. Starace

University of Nebraska-Lincoln, astarace1@unl.edu

Follow this and additional works at: https://digitalcommons.unl.edu/physicsfacpub

Part of the Physics Commons

Krylovetsky, A. A.; Manakov, N. L.; Marmo, S. I.; and Starace, Anthony F., "Circular dichroism in classical Coulomb scattering involving bremsstrahlung" (2005). Faculty Publications, Department of Physics and Astronomy. 15.

https://digitalcommons.unl.edu/physicsfacpub/15

This Article is brought to you for free and open access by the Research Papers in Physics and Astronomy at DigitalCommons@University of Nebraska - Lincoln. It has been accepted for inclusion in Faculty Publications, Department of Physics and Astronomy by an authorized administrator of DigitalCommons@University of Nebraska Lincoln. 


\title{
Circular dichroism in classical Coulomb scattering involving bremsstrahlung
}

\author{
A. A. Krylovetsky, ${ }^{1}$ N. L. Manakov, ${ }^{1}$ S. I. Marmo, ${ }^{1}$ and Anthony F. Starace ${ }^{2}$ \\ ${ }^{1}$ Department of Physics, Voronezh State University, 394006, Voronezh, Russia \\ ${ }^{2}$ Department of Physics and Astronomy, The University of Nebraska, Lincoln, Nebraska 68588-0111, USA
}

(Received 17 June 2005; published 16 September 2005)

\begin{abstract}
Classical orbit derivations of the triply differential cross section for electron bremsstrahlung $(\mathrm{BrS})$ in a Coulomb field are presented; the classical origin of the circular dichroism effect in the electron scattering angular distributions is established for both spontaneous and laser-stimulated $\mathrm{BrS}$.
\end{abstract}

DOI: 10.1103/PhysRevA.72.035401

PACS number(s): 34.80.Qb, 41.60.-m

Nonrelativistic dipole bremsstrahlung (BrS) emission by an electron scattered from a centrally symmetric (e.g., Coulomb) potential is a fundamental radiative process that allows one to analyze quantum-classical correspondences within radiation theory. Even now, at the centenary of the quantum theory, classical analyses remain useful for understanding fine details of the BrS process (see, e.g., Ref. [1]). Since the first classical electrodynamics analysis by Kramers [2], exact analytical results for the Coulomb BrS cross section have been obtained both quantum mechanically [3] and classically [4]. The quantum results provide the most complete information on the $\mathrm{BrS}$ process via the triply differential cross section

$$
\sigma^{(3)} \equiv \frac{d \sigma\left(\mathbf{e}, \mathbf{k} ; \mathbf{p}, \mathbf{p}^{\prime}\right)}{d \omega d \Omega_{\mathbf{p}^{\prime}} d \Omega_{\mathbf{k}}}
$$

for emission of a photon [of frequency $\omega$, polarization vector $\mathbf{e}\left(\mathbf{e} \cdot \mathbf{e}^{*}=1\right)$ and wave vector $\left.\mathbf{k}(\mathbf{k}=(\omega / c) \hat{\mathbf{k}})\right]$ by an incident electron with momentum $\mathbf{p}$ that is scattered in the direction $\hat{\mathbf{p}}^{\prime}\left(\right.$ where $\mathbf{p}^{\prime}=p^{\prime} \hat{\mathbf{p}}^{\prime}$ and $\left.p^{\prime}=\sqrt{p^{2}-2 m \hbar \omega}\right)$.

In the classical theory, the key dynamics of the BrS process summed over the polarization of the radiation or integrated over the directions $\hat{\mathbf{p}}^{\prime}$ of the scattered electrons has been analyzed in detail and compared with the corresponding quantum results. For example, the spectral density of $\mathrm{BrS}$ radiation $d \kappa_{\omega} / d \omega$ can be expressed in terms of the energy emitted by a charged particle at fixed impact parameter $\rho$, $d \mathcal{E}_{\omega}(\rho) / d \omega$, as follows [4]:

$$
\frac{d \kappa_{\omega}}{d \omega}=\int_{0}^{\infty} \frac{d \mathcal{E}_{\omega}(\rho)}{d \omega} 2 \pi \rho d \rho \equiv \hbar \omega \frac{d \sigma_{c l}(\omega)}{d \omega},
$$

where $d \sigma_{c l}(\omega) / d \omega$ is the classical counterpart of the quantum singly differential cross section $d \sigma(\omega) / d \omega$. For spontaneous $\mathrm{BrS}$, the measurement of the scattered electron angular distributions and their dependence on the polarization of the $\mathrm{BrS}$ radiation is possible only in coincidence experiments (with polarization-sensitive photodetectors) and is thus difficult. However, the current experimental ability to vary the laser light polarization raises interest in the investigation of polarization effects in laser-stimulated radiative collisions. The simplest ones are stimulated $\mathrm{BrS}(\mathrm{SBrS})$ and inverse $\mathrm{BrS}$ processes for elastic electron-atom scattering in the presence of a laser field. For a low-intensity laser field, the high- intensity corrections to the one-photon $\mathrm{SBrS}$ cross section as well as multiphoton $\mathrm{SBrS}$ processes are negligible, so that the $\mathrm{SBrS}$ cross section is closely related to that for spontaneous BrS [see Eq. (19) below].

We present here the classical result, $\sigma_{c l}^{(3)}$, for the triply differential cross section of polarized $\mathrm{BrS}$ radiation originating from low-energy electron scattering from a Coulomb center of charge, $Z|e|$ [i.e., the classical counterpart of Eq. (1)]. Our analysis shows that the difference in the cross sections for electron scattering in the fixed direction $\mathbf{p}^{\prime}$ involving the emission of left-hand- or right-hand-polarized spontaneous $\mathrm{BrS}$ or $\mathrm{SBrS}$ radiation in the direction $\mathbf{k}$ [the circular dichroism (CD) effect], which in the quantum treatment appears as the result of quantum interferences [5], exists also in the results for $\sigma_{c l}^{(3)}$, thus showing the classical origin of the CD effect.

To treat polarized dipole radiation emitted by an electron moving along a classical orbit, we use the Hamiltonian formalism in the classical theory of radiation [6], which has been adapted in Ref. [7] for the analysis of BrS processes within the framework of "Kramers electrodynamics." In this approach, the electromagnetic field in the volume $V=L^{3}$ is described by a set of field oscillators, whose generalized coordinates $q_{\mathbf{e}_{i}, \mathbf{k}_{i}}(t)$ correspond to the monochromatic planewave modes $\mathbf{A}_{\mathbf{e}_{i}, \mathbf{k}_{i}}(\mathbf{r})$ of the vector potential at the position $\mathbf{r}$. For the mode corresponding to radiation with polarization vector $\mathbf{e}$ and wave vector $\mathbf{k}$, the equation for $q_{\mathbf{e}_{i}, \mathbf{k}_{i}}(t)$ is (cf. [7])

$$
\ddot{q}_{\mathbf{e}, \mathbf{k}}+\omega^{2} q_{\mathbf{e}, \mathbf{k}}=\sqrt{4 \pi / V} e(\mathbf{e} \cdot \mathbf{v}(t)) \exp [i \mathbf{k} \cdot \mathbf{r}(t)],
$$

where $\mathbf{r}(t)$ and $\mathbf{v}(t)=\dot{\mathbf{r}}(t)$ are the position vector and velocity of an electron on a prescribed classical orbit. Equation (3) represents a forced vibration of an oscillator due to the motion of a charged particle. The energy emitted by the field oscillator in the $(\mathbf{k}, \mathbf{e})$ mode is the energy transferred from the electron to the oscillator. This energy equals the work $W$ done by the "external force" on the right-hand side (RHS) of Eq. (3); it may be expressed in terms of the Fourier transform of the RHS of Eq. (3) as a function of the frequency $\omega$ [8]. This Fourier component is proportional to that of the classical velocity $\mathbf{v}(t), \mathbf{v}_{-\omega}=\int_{-\infty}^{\infty} \mathbf{v}(t) \exp (-i \omega t) d t$ note that $\left(\mathbf{v}_{\omega}=\mathbf{v}_{-\omega}{ }^{*}\right)$, assuming the electron motion is nonrelativistic and thus that the electric-dipole approximation $\exp [i \mathbf{k} \cdot \mathbf{r}(t)]$ $\approx 1$ may be used on the RHS of Eq. (3). Thus, $W$ 
$=(2 \pi / V) e^{2}\left|\mathbf{e} \cdot \mathbf{v}_{-\omega}\right|^{2}$. Multiplying $W$ by the number of field oscillators in the volume $V$ and in the element $d \mathbf{k}$ of $\mathbf{k}$ space, $\left(V \omega^{2} d \omega d \Omega_{\mathbf{k}}\right) /(2 \pi c)^{3}$, the energy $d \mathcal{E}_{\mathbf{e} \omega}$ of polarized radiation emitted in the frequency interval $d \omega$ within the solid angle $d \Omega_{\mathbf{k}}$ by an electron moving along a trajectory $\mathbf{r}(t)$ is

$$
d \mathcal{E}_{\mathbf{e} \omega}=\frac{e^{2} \omega^{2}}{4 \pi^{2} c^{3}}\left|\mathbf{e}^{*} \cdot \mathbf{v}_{\omega}\right|^{2} d \omega d \Omega_{\mathbf{k}} .
$$

This result generalizes the classical result for the energy $d \mathcal{E}_{\omega}$ of dipole radiation with frequency in the interval $d \omega$ (emitted during the entire time of collision) to the case of a specified polarization state (e) of the radiation. Summing Eq. (4) over the polarization states and integrating over the direction of $\mathbf{k}$, which is equivalent to the substitution [9]

$$
e_{i} e_{j}^{*} d \Omega_{\mathbf{k}} \rightarrow(8 \pi / 3) \delta_{i j},
$$

one obtains the known result [4]

$$
d \mathcal{E}_{\omega}=\frac{2 e^{2} \omega^{2}}{3 \pi c^{3}}\left|\mathbf{v}_{\omega}\right|^{2} d \omega=\frac{2 \omega^{4}}{3 \pi c^{3}}\left|\mathbf{d}_{\omega}\right|^{2} d \omega .
$$

For an electron moving along a hyperbolic trajectory in a Coulomb field, the Cartesian coordinates of $\mathbf{v}_{\omega}, \dot{x}_{\omega}$, and $\dot{y}_{\omega}$, in the orbit plane $(x, y)$ (with the $x$ axis chosen as the symmetry axis of the trajectory) are well known [4]:

$$
\begin{gathered}
\dot{x}_{\omega}=i \frac{2 Z e^{2}}{m v^{2}} e^{\pi \nu / 2} K_{i \nu}^{\prime}(\nu \varepsilon), \\
\dot{y}_{\omega}=\frac{2 Z e^{2}}{m v^{2}} \frac{\sqrt{\varepsilon^{2}-1}}{\varepsilon} e^{\pi \nu / 2} K_{i \nu}(\nu \varepsilon),
\end{gathered}
$$

where $K_{\mu}(z)$ and $K_{\mu}^{\prime}(z)$ are the Macdonald function and its derivative with respect to $z$, and $\nu$ and $\varepsilon$ are a dimensionless "classical frequency" and an angular parameter,

$$
\nu=\frac{Z e^{2} \omega}{m v^{3}}, \quad \varepsilon=\frac{1}{\sin (\theta / 2)},
$$

where $\theta$ is the scattering angle and $\cos \theta=\left(\hat{\mathbf{p}} \cdot \hat{\mathbf{p}}^{\prime}\right)$. Note that $K_{\mu}(z)$ is related to the Hankel function of imaginary argument, $H_{\mu}^{(1)}(i z)[10]: K_{\mu}(z)=i(\pi / 2) \exp (i \pi \mu / 2) H_{\mu}^{(1)}(i z)$. Also, $p^{\prime}=p=m v$ since the damping effect of $\mathrm{BrS}$ radiation on the electron motion is neglected in a classical treatment. The vector $\mathbf{v}_{\omega}=\dot{x}_{\omega} \hat{x}+\dot{y}_{\omega} \hat{y}$ may be expressed in an invariant form

$$
\mathbf{v}_{\omega}=i \varepsilon \frac{Z e^{2}}{m v^{2}} e^{\pi \nu / 2}\left[\frac{i}{\varepsilon} K_{i \nu}(\nu \varepsilon) \mathbf{p}_{+}+K_{i \nu}^{\prime}(\nu \varepsilon) \mathbf{p}_{-}\right],
$$

where we have represented the unit vectors $\hat{x}$ and $\hat{y}$ in terms of the vectors $\mathbf{p}_{ \pm}=\hat{\mathbf{p}}^{\prime} \pm \hat{\mathbf{p}}$

$$
\hat{x}=\frac{\varepsilon}{2} \mathbf{p}_{-}, \quad \hat{y}=-\frac{\varepsilon}{2 \sqrt{\varepsilon^{2}-1}} \mathbf{p}_{+} .
$$

The use of recurrence relations for the Macdonald functions leads to an alternative representation for $\mathbf{v}_{\omega}$ in terms of a single (complex) Macdonald function:

$$
\mathbf{v}_{\omega}=i \varepsilon \frac{Z e^{2}}{m v^{2}} e^{\pi \nu / 2}\left[K_{1+i \nu}(\nu \varepsilon) \hat{\mathbf{p}}-K_{1+i \nu}^{*}(\nu \varepsilon) \hat{\mathbf{p}}^{\prime}\right]
$$

To introduce the classical triply differential cross section $\sigma_{c l}^{(3)}$, the energy $d \mathcal{E}_{\mathrm{e} \omega}$ emitted by an electron on a single trajectory [from Eq. (4)] should be multiplied by the element $d S=\rho d \rho d \phi$ in the (orthogonal to $\mathbf{p}$ ) plane of impact parameters. For a given classical orbit in a Coulomb field, the relation between $d S$ and the corresponding scattered electron solid angle element $d \Omega_{\mathbf{p}^{\prime}}$ is

$$
\rho d \rho d \phi=\widetilde{\sigma}_{0} d \Omega_{\mathbf{p}^{\prime}},
$$

where

$$
\widetilde{\sigma}_{0} \equiv \frac{d \sigma_{0}}{d \Omega_{\mathbf{p}^{\prime}}}=\left(\frac{Z e^{2}}{2 m v^{2}}\right)^{2} \varepsilon^{4}
$$

is the Rutherford cross section. Thus, $\sigma_{c l}^{(3)}$ is given by the product of the right-hand sides of Eqs. (4) and (12) divided by $\hbar \omega d \omega d \Omega_{\mathbf{p}^{\prime}} d \Omega_{\mathbf{k}}$. Using Eq. (11), $\sigma_{c l}^{(3)}$ is thus

$$
\begin{aligned}
\sigma_{c l}^{(3)}= & \widetilde{\sigma}_{0} \frac{\alpha}{4 \pi^{2}} \frac{v^{2}}{c^{2}} \frac{\nu^{2} \varepsilon^{2}}{\omega} e^{\pi \nu} \\
& \times\left|K_{1+i \nu}(\nu \varepsilon)\left(\mathbf{e}^{*} \cdot \hat{\mathbf{p}}\right)-K_{1-i \nu}(\nu \varepsilon)\left(\mathbf{e}^{*} \cdot \hat{\mathbf{p}}^{\prime}\right)\right|^{2},
\end{aligned}
$$

where $\alpha=e^{2} /(\hbar c)$. Substituting Eq. (9) into Eq. (6), one obtains the known results for the energy $d \mathcal{E}_{\omega}(\rho)$ and spectral density $d \kappa_{\omega} / d \omega$ of Coulomb BrS [4]. Also, using Eqs. (4) and (9) to calculate the differential spectral density $d \kappa_{\mathbf{k} \omega} / d \omega$, the angular distributions of Coulomb $\mathrm{BrS}$ radiation (with respect to the vector $\mathbf{p}), d \kappa_{\mathbf{k} \omega}^{\|} / d \omega$ and $d \kappa_{\mathbf{k} \omega}^{\perp} / d \omega$, polarized, respectively, in the plane $(\mathbf{p}, \mathbf{k})$ and that orthogonal to $(\mathbf{p}, \mathbf{k})$, have the known form of integrals of Macdonald (or Hankel) functions. [In Ref. [11] these integrals are calculated analytically in terms of $K_{i \nu}(\nu), K_{i \nu}^{\prime}(\nu), d K_{\mu}(\nu) / d \mu$, and $d K_{\mu}^{\prime}(\nu) / d \mu$ (with $\mu=i \nu$ ); in Ref. [12] they are calculated in terms of generalized hypergeometric functions ${ }_{p} F_{p+1}$, with $p=1,2,3$.]

The classical cross section $\sigma_{c l}^{(3)}$ in Eq. (14) should be compared with the quantum result [3,9], which we present here in a similar form

$$
\begin{aligned}
\sigma^{(3)}= & \alpha\left(\frac{2 Z e^{2}}{m c}\right)^{2} \frac{a a^{\prime} v^{\prime}}{\omega v\left(v-v^{\prime}\right)^{4}\left(e^{2 \pi a}-1\right)\left(1-e^{-2 \pi a^{\prime}}\right)} \\
& \times\left|Q\left(v, v^{\prime} ; \varepsilon\right)\left(\mathbf{e}^{*} \cdot \hat{\mathbf{p}}\right)-Q\left(v^{\prime}, v ; \varepsilon\right)\left(\mathbf{e}^{*} \cdot \hat{\mathbf{p}}^{\prime}\right)\right|^{2} .
\end{aligned}
$$

The amplitude $Q$ contains the Gauss hypergeometric function ${ }_{2} F_{1}(a, b ; c ; z)$,

$$
Q\left(v, v^{\prime} ; \varepsilon\right)=v(1-i a)_{2} F_{1}\left(2-i a, 1-i a^{\prime} ; 2 ; \lambda_{0}\right),
$$

where

$$
a=\frac{Z e^{2}}{\hbar v}, \quad a^{\prime}=\frac{Z e^{2}}{\hbar v^{\prime}}, \quad \lambda_{0}=-\frac{4 v v^{\prime}}{\left(v-v^{\prime}\right)^{2} \varepsilon^{2}} .
$$

The quantum result (15) reduces to the classical one (14) when $\hbar \rightarrow 0$. Asymptotic forms of the functions ${ }_{2} F_{1}$ for large parameters $a$ and $a^{\prime}$ [with $\left(a^{\prime}-a\right)$ remaining finite] were obtained in analyses of quantum corrections to the classical orbit result for proton $\mathrm{BrS}$ [13]. In our case, the asymptotic form of ${ }_{2} F_{1}$ in Eq. (16) is 


$$
\begin{aligned}
& { }_{2} F_{1}\left(2-i a, 1-i a^{\prime} ; 2 ; \lambda_{0}\right) \\
& \quad \approx \frac{i}{\pi a} e^{(\pi / 2)\left(a+a^{\prime}\right)-2 i \nu}(\nu \varepsilon / 2 a)^{3-i a-i a^{\prime}} K_{1+i \nu}(\nu \varepsilon) .
\end{aligned}
$$

Using Eq. (18) and taking into account the relations

$$
\left(v-v^{\prime}\right) \rightarrow(\hbar \omega) /(m v), \quad\left(a^{\prime}-a\right) \rightarrow \nu,
$$

Eq. (15) reduces to Eq. (14). [For the case of linear polarization $\left(\mathbf{e}=\mathbf{e}^{*}\right)$, Eq. (14) for $\sigma_{c l}^{(3)}$ may be deduced also from the semiclassical results of Ref. [11] for $n$-photon SBrS for the case of $n=1$ and a low-intensity laser field. However, our analysis shows that already for $n=2$, the low-intensity result of Ref. [11] does not coincide with the classical limit ( $\hbar$ $\rightarrow 0$ ) of the exact quantum results for two-photon $\mathrm{BrS}$ (as will be shown elsewhere).]

The relation between $\sigma^{(3)}$ and the cross section, $d \sigma / d \Omega_{\mathbf{p}^{\prime}}$, for $\mathrm{SBrS}$ emission in laser-assisted electron scattering is given by

$$
\frac{d \sigma}{d \Omega_{\mathbf{p}^{\prime}}}=\frac{\pi^{2} c^{3}}{\hbar \omega^{3}} F^{2} \sigma^{(3)},
$$

where $F$ is the amplitude of a laser field having an electric vector $\mathbf{F}(t)=F \operatorname{Re}\{\mathbf{e} \exp [i(\mathbf{k} \cdot \mathbf{r}-\omega t)]\}$ and an intensity $I$ $=c F^{2} /(8 \pi)$. Thus, $\sigma_{c l}^{(3)}$ allows one to analyze polarization effects in both stimulated and spontaneous classical BrS. The most interesting of these effects is the CD effect in the angular distributions of scattered electrons. (For a general discussion of circular and elliptic dichroism effects in various atomic photoprocesses see Ref. [14].) The polarization vector $\mathbf{e}$ is generally complex (for an elliptical polarization). Taking into account the identity [5]

$$
2 \operatorname{Im}\left\{\left(\mathbf{e}^{*} \cdot \hat{\mathbf{p}}\right)\left(\mathbf{e} \cdot \hat{\mathbf{p}}^{\prime}\right)\right\}=\xi\left(\hat{\mathbf{k}} \cdot\left[\hat{\mathbf{p}} \times \hat{\mathbf{p}}^{\prime}\right]\right), \quad-1 \leqslant \xi \leqslant 1,
$$

where the pseudoscalar $\xi$ is the degree of circular polarization (or the Stokes parameter $\xi_{2}$ ), $\xi=i\left(\hat{\mathbf{k}} \cdot\left[\mathbf{e} \times \mathbf{e}^{*}\right]\right.$ ) (cf. Sec. 8 in Ref. [9]), and using Eq. (9) for $\mathbf{v}_{\omega}$, we find

$$
\begin{aligned}
\sigma_{c l}^{(3)}= & \widetilde{\sigma}_{0} \frac{\alpha}{4 \pi^{2}} \frac{v^{2}}{c^{2}} \frac{\nu^{2}}{\omega} e^{\pi \nu}\left\{K_{i \nu}^{2}(\nu \varepsilon)\left|\mathbf{e}^{*} \cdot \mathbf{p}_{+}\right|^{2}+\varepsilon^{2} K_{i \nu}^{\prime 2}(\nu \varepsilon)\left|\mathbf{e}^{*} \cdot \mathbf{p}_{-}\right|^{2}\right. \\
& \left.-2 \xi \varepsilon K_{i \nu}(\nu \varepsilon) K_{i \nu}^{\prime}(\nu \varepsilon)\left(\hat{\mathbf{k}} \cdot\left[\hat{\mathbf{p}} \times \hat{\mathbf{p}}^{\prime}\right]\right)\right\}
\end{aligned}
$$

One sees that $\sigma_{c l}^{(3)}$ is different for opposite helicities of the $\mathrm{BrS}$ radiation (i.e., upon substituting $\xi \rightarrow-\xi$ or $\mathbf{e} \rightarrow \mathbf{e}^{*}$ ). For both classical and quantum cross sections, the "CD difference" $\Delta_{C D}^{c l} \equiv \sigma_{c l}^{(3)}(\mathbf{e})-\sigma_{c l}^{(3)}\left(\mathbf{e}^{*}\right)$ has a kinematical maximum when $\mathbf{p} \perp \mathbf{p}^{\prime}$ and the polarization plane coincides with the scattering plane (cf. Fig. 1). In the quantum approach, the $\mathrm{CD}$ effect originates from the interference between real and imaginary parts of the complex $\mathrm{BrS}$ amplitudes $Q\left(v, v^{\prime}, \varepsilon\right)$ and $Q\left(v^{\prime}, v, \varepsilon\right)$ in Eq. (15) [5]. Equation (21) shows that in the classical treatment the CD effect originates from interference of the real and imaginary "classical amplitudes" $K_{i \nu}^{\prime}(\nu \varepsilon)$ and $(i / \varepsilon) K_{i \nu}(\nu \varepsilon)$ in Eq. (9). [Note that both $K_{i \nu}(\nu \varepsilon)$ and $K_{i \nu}^{\prime}(\nu \varepsilon)$ are real.] This classical CD effect shows that the emission of polarized $\mathrm{BrS}$ radiation is sensitive not only to

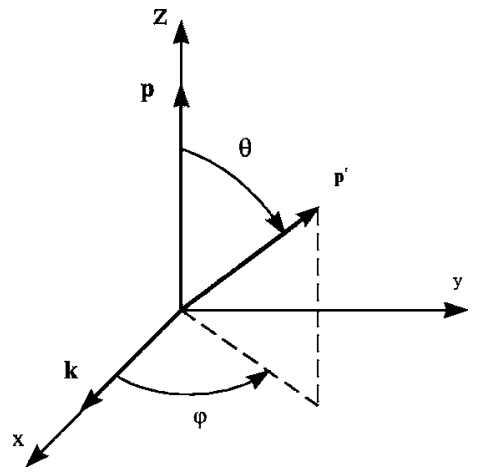

FIG. 1. Geometry of an angularly resolved BrS process.

the mutual orientation of the classical orbit plane and the wave vector $\mathbf{k}$ (i.e., the polarization plane), but also to the handedness of the BrS radiation.

The CD effect vanishes for forward and backward scattering $\left(\mathbf{p}^{\prime}= \pm \mathbf{p}\right)$, for $\mathrm{BrS}$ emission in the scattering plane $\left(\mathbf{k} \perp\left[\hat{\mathbf{p}} \times \hat{\mathbf{p}}^{\prime}\right]\right)$, and also when the cross section is integrated over the direction of the scattered electron's momentum, $\mathbf{p}^{\prime}$. It becomes small in the low-frequency domain $(\nu \varepsilon \ll 1)$, where $\sigma_{c l}^{(3)}$ has the following form:

$$
\begin{aligned}
\sigma_{c l}^{(3)} \approx & \widetilde{\sigma}_{0} \frac{\alpha}{4 \pi^{2}} \frac{v^{2}}{c^{2} \omega} e^{\pi \nu} \\
& \times\left[\left|\mathbf{e}^{*} \cdot \mathbf{p}_{-}\right|^{2}+2 \xi \nu \ln \frac{2}{\gamma \nu \varepsilon}\left(\hat{\mathbf{k}} \cdot\left[\hat{\mathbf{p}} \times \hat{\mathbf{p}}^{\prime}\right]\right)\right]
\end{aligned}
$$

where $\gamma=1.781 \ldots$ is the Euler constant. In the highfrequency limit $(\nu \gg 1)$, the CD term in $\sigma_{c l}^{(3)}$ has no small parameters, as may be seen from the asymptotic result for $\sigma_{c l}^{(3)}$ for $\nu \gg 1$ and $\theta<\pi$ :

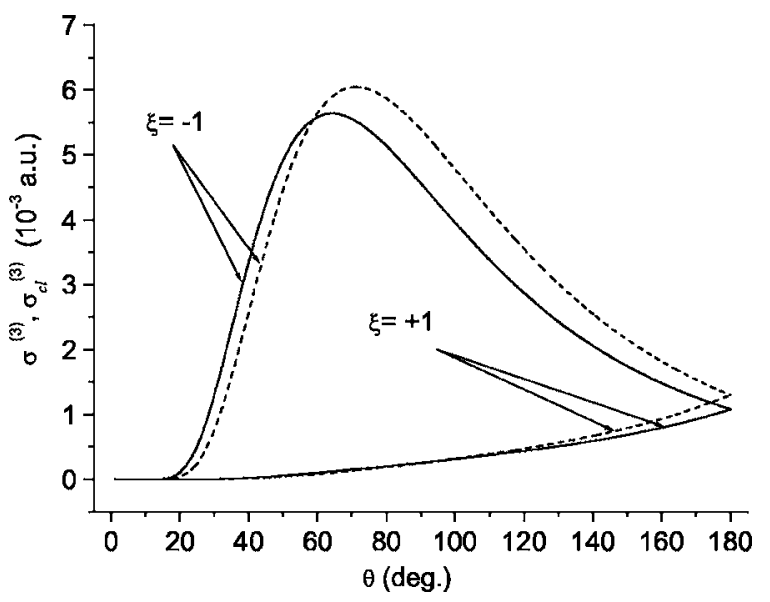

FIG. 2. Comparison of classical and quantum triply differential cross sections $\sigma_{c l}^{(3)}$ (solid curves) and $\sigma^{(3)}$ (dashed curves) as functions of the electron scattering angle $\theta$ for left- (top curves) and right- (bottom curves) circular polarizations, $\xi=\mp 1$ of the emitted $\mathrm{BrS}$ radiation. For all curves, $Z / p=10$ a.u., $\nu=1$ (i.e., $\hbar \omega$ $=10^{-3} Z^{2}$ a.u.), and $\varphi=\pi / 2$. 


$$
\begin{aligned}
\sigma_{c l}^{(3)} \approx & \widetilde{\sigma}_{0} \frac{\alpha}{8 \pi} \frac{v^{2}}{c^{2}} \frac{\nu \varepsilon^{2}}{\omega} \tan \frac{\theta}{2} e^{-\nu[\theta+2 \cot (\theta / 2)-\pi]} \\
& \times\left\{\sin ^{2}(\theta / 2)\left|\mathbf{e}^{*} \cdot \mathbf{p}_{+}\right|^{2}+\cos ^{2}(\theta / 2)\left|\mathbf{e}^{*} \cdot \mathbf{p}_{-}\right|^{2}\right. \\
& \left.+\xi \sin \theta\left(\hat{\mathbf{k}} \cdot\left[\hat{\mathbf{p}} \times \hat{\mathbf{p}}^{\prime}\right]\right)\right\} .
\end{aligned}
$$

[Equations (22) and (23) follow from Eq. (21) using the limiting forms of the Macdonald functions.]

A significant $\mathrm{CD}$ effect beyond the low-frequency domain (i.e., for finite $\nu$ ) is illustrated in Fig. 2 for the geometry shown in Fig. 1. One sees that the classical and quantum results are in good qualitative agreement and that in both cases the CD difference between cross sections for opposite helicities of the emitted $\mathrm{BrS}$ radiation can be as large as an order of magnitude. Figure 3 shows that the dependence of the ratio $\sigma_{c l}^{(3)} / \sigma^{(3)}$ on the Sommerfeld parameter $a$ $=\left(Z e^{2}\right) /(\hbar v)(a=Z / p$ in a.u. $)$ exhibits the typical decreasing behavior with increasing $p$ known from earlier studies of total and differential (in k) BrS cross sections. The CD difference in the classical cross section $\sigma_{c l}^{(3)}$ (see the inset in Fig. 3 ) decreases with increasing $p$ in qualitative agreement with the known fact that the CD effect disappears in the Born approximation [5].

In conclusion, we have obtained the classical result for the triply differential cross section for Coulomb $\mathrm{BrS}$ and have demonstrated that the $\mathrm{CD}$ effects in the angular distributions

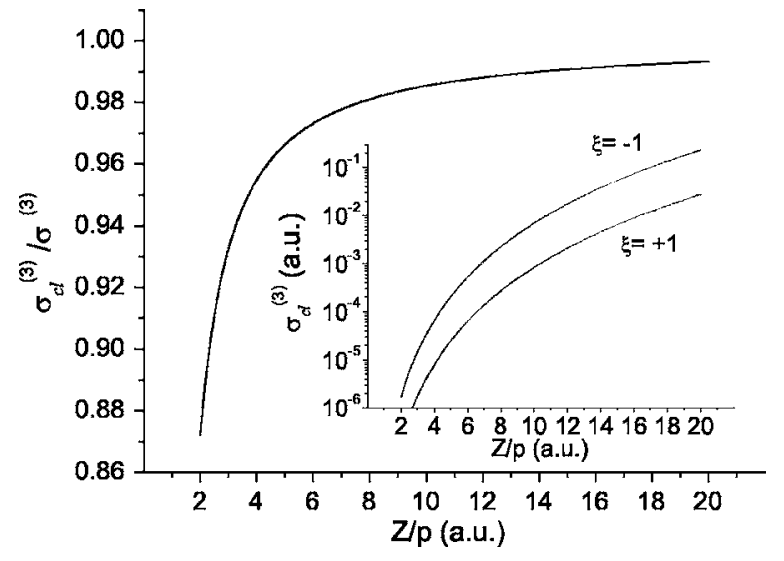

FIG. 3. Dependence of the ratio $\sigma_{c l}^{(3)} / \sigma^{(3)}($ for $\xi=+1)$ and, in the inset, $\sigma_{c l}^{(3)}($ for $\xi= \pm 1)$ on $a=Z / p$, for $\theta=\pi / 2, \varphi=\pi / 2$, and $\nu=0.5$ [i.e., $\hbar \omega=p^{3} /(2 Z)$ a.u.].

of the scattered electrons in both stimulated and spontaneous $\mathrm{BrS}$ processes have the same qualitative features as in the corresponding quantum results.

This work was supported in part by NSF Grant No. PHY0300665, RFBR Grant No. 04-02-16350, and by the BRHE Program of U.S. CRDF \& Russian Ministry of Education and Science, Grants Nos. VZ-010 and Y2-P-10-09 (A.A.K.).
[1] A. Florescu, O. I. Obolensky, and R. H. Pratt, J. Phys. B 35, 2911 (2002).

[2] H. A. Kramers, Philos. Mag. 46, 836 (1923).

[3] A. Sommerfeld, Ann. Phys. (Leipzig) 11, 257 (1931); Wellenmechanik (Frederick Ungar, New York, 1950), Chap. 7.

[4] L. D. Landau and E. M. Lifshitz, The Classical Theory of Fields, 4th ed. (Pergamon Press, Oxford, 1975), Chap. IX.

[5] N. L. Manakov, S. I. Marmo, and V. V. Volovich, Phys. Lett. A 204, 42 (1995); N. L. Manakov, S. I. Marmo, and V. V. Volovich, J. Electron Spectrosc. Relat. Phenom. 79, 327 (1996).

[6] W. Heitler, The Quantum Theory of Radiation, 3rd ed. (Clarendon, Oxford, 1954), Sec. I.6.

[7] V. I. Kogan, A. B. Kukushkin, and V. S. Lisitsa, Phys. Rep. 213, 1 (1992), Sec. 1.1.

[8] L. D. Landau and E. M. Lifshitz, Mechanics, 3rd ed. (Perga- mon Press, Oxford, 1976), Sec. 22.

[9] V. B. Berestetsky, E. M. Lifshitz, and L. P. Pitaevsky, Quantum Electrodynamics, 2nd ed. (Pergamon Press, Oxford, 1982), Sec. 92.

[10] G. N. Watson, A Treatise on the Theory of Bessel Functions, 2nd ed. (Cambridge University Press, Cambridge, England, 1944), Sec. 3.7.

[11] I. Ya. Berson, Zh. Eksp. Teor. Fiz. 80, 1727 (1981) [Sov. Phys. JETP 53, 891 (1981)].

[12] V. Florescu, V. Steiner, and L. Burlacu, J. Phys. B 20, 427 (1987).

[13] S. D. Drell and K. Huang, Phys. Rev. 99, 686 (1955).

[14] N. L. Manakov, M. V. Frolov, and A. F. Starace, J. Phys. B 35, R49 (2003), Sec. 1.1. 